\title{
A Novel Probiotic Strain Exerts Therapeutic Effects on Mouse Model of Multiple Sclerosis by Altering The Expression of Inflammasome and IDO Genes and Modulation of T Helper Cytokine Profile
}

saba sadeghi ( $\nabla$ sadeghi.saba@yahoo.com )

Islamic Azad University Central Tehran Branch

fatemeh kazemi

Islamic Azad University Central Tehran Branch

saba taheri

Islamic Azad University Islamshahr Branch: Islamic Azad University Eslamshahr Branch

Maryam Tajabadi Ebrahimi

Islamic Azad University Central Tehran Branch

javad arasteh

Islamic Azad University Central Tehran Branch https://orcid.org/0000-0002-1564-7494

\section{Research Article}

Keywords: Multiple sclerosis, Inflammasome, Inflammation, Bacillus coagulans, Cuprizone

Posted Date: July 6th, 2021

DOI: https://doi.org/10.21203/rs.3.rs-584482/v1

License: (c) (1) This work is licensed under a Creative Commons Attribution 4.0 International License.

Read Full License 


\section{Abstract}

Multiple sclerosis is an inflammatory demyelinating disease that commences to neuronal cell destruction. Recently, a promising evidence of synergic effects of combined supplementation with vitamin $\mathrm{D}$ and probiotics in modulating the gut microbiota and metabolome is emerging. Bacillus Coagulans IBRC-M10791 as a novel strain was chosen, prevention and treatment impacts of regular administered were studied in Cuprizone-induced C57bl/ 6 mouse of demyelination. The mice were divided into six groups and received a daily dose of cuprizone or probiotics. To investigate the effect of probiotic, the IDO-1, CYP27B1, NLRP1, NLRP3, and AIM2 expression were estimated by Real-Time PCR, and IL-4, IL17, IFN-gamma, and TGF-beta cytokines were measured by ELISA. The results showed that there was significant decrease in IL-17 and IFN- $\gamma$ and modulatory effects on IL-4 and TGF- $\beta$. On the other hand, we demonstrated that there are significant decrease for expression of ID 0-1, CYP27b1, NLRP1, NLRP3 and AIM2 genes in prevention and treatment groups compared to cuprizone group. Also, a significant enhancement in rate of remyelination and alternations proved by LFB staining and Y-Maze test. In conclusion, our study provides insight into how the therapeutic effect of the chosen strain of probiotic was correlated with the modulation of the level of inflammatory and anti-inflammatory cytokines. Further, we demonstrated that the expression of genes related to Tryptophan, Vitamin D and Inflammasome pathways could be affected by Bacillus coagulans. Our study could be beneficial to provide a novel Cotherapeutic strategy for Multiple sclerosis.

\section{Introduction}

Multiple sclerosis is an autoimmune, inflammatory disease, and one of the most common diseases of the central nervous system that most often occurs in the age range of 20 to 40 years(Schattling et al. 2014). Although the cause of MS remains unknown, several factors such as genetics, environment, vitamin D, and immune system are involved in the occurrence of this disease(Goodin 2016), but it is eventually diagnosed with the loss of myelin, nerve damage, and the development of neurological disabilities(Bar-Or et al. 2011).

Various studies on patients with MS indicate that multiple aspects of the innate and adaptive immune systems do not operate correctly in these patients(Høglund and Maghazachi 2014). In adaptive immune system, excessive increase in invasive activity of Th1 and Th17 by secreting the pro-inflammatory cytokines IFN- $y$ and IL-17, respectively, plays a crucial role in the immunopathogenesis of MS as well as modulation of anti-inflammatory cytokines such as IL-4 and TGF- $\beta$ through Th2 and Treg cells(Duhen et al. 2013). On the other hand, IFN- $y$ expression increases transcription of the IDO gene, resulting in decreased $T$ cell activation and proliferation through tryptophan depletion and production of Treg lymphocytes(Lee and Mazmanian 2010). Researches have shown that this gene is involved in the pathogenesis of autoimmune diseases, and a change in its expression could indicate the onset of relapse in MS patients(Lovelace et al. 2016). The role of the innate immune system in MS has recently received much attention. Inflammasome complex as part of this system detect inflammatory signals generated by infection or endogenous and with increasing the expression of genes such as NLRP1, NLRP3, and AIM2 
cause cell maturation and production of IL-1 $\beta$ and IL-18 inflammatory cytokines as well as stimulating the IFN- $\gamma$ (Govindarajan et al. 2020). Previously the gastrointestinal tract was considered the only place to digest and absorb nutrients, while today, several functions such as affecting the body and the nervous system's general health are recognized(Yu et al. 2016). In the gastrointestinal tract, vital bacteria are called intestinal flora that is metabolically active and involved in the production of vitamins and neurotransmitters(Usami et al. 2015) and influenced by factors such as age, genetics, race, host nutrition, geography, stressors and they can be changed by taking probiotics(Hughes 2020). Various studies have reported some therapeutic potential of probiotics in reducing the clinical symptoms of many gastrointestinal diseases, improving allergies, and enhancement of the immune system(Yan and Polk 2011). Numerous studies have also shown that probiotics can reduce inflammation by integrating the barrier of the gastrointestinal tract and reduces the leaking ability of endotoxins into the bloodstream(König et al. 2016). The complex interaction between the host immune system and the normal intestinal flora is essential for maintaining intestinal homeostasis. If dysbiosis happens as a result of changes in the function and type of bacteria, the natural flora can cause or spread autoimmune and infectious diseases(Belkaid and Hand 2014). Recently, a promising evidence of synergic effects of combined supplementation with vitamin $\mathrm{D}$ and probiotics in modulating the gut microbiota and metabolome is emerging as well as a correlation between the level of serum vitamin D and MS risk and disease activity. These effects are through improving the expression of vitamin D receptors. Therefore, modulating the microbiota-gut-brain axis by probiotics plus improving vitamin $\mathrm{D}$ levels might provide a novel target to treat mental and metabolic disorders(Ostadmohammadi et al. 2019). Vitamin D are hydroxylated by the cytochrome P450 enzyme CYP27A1 in the liver to 25-hydroxyvitamin D (25[OH]D) in substrate-dependent reaction then it circulates in the blood bound to the vitamin D-binding protein and is a reliable indicator of vitamin $D$ status. To become fully activated, the $25(\mathrm{OH}) \mathrm{D}$ is converted into 1,25 dihydroxyvitamin $D(1,25[\mathrm{OH}] 2 \mathrm{D})$ by the mitochondrial 1 a-hydroxylase enzyme (CYP27B1). The majority of the body's 1,25(OH)2D is synthesized in the primary renal tubules of the kidney, but synthesis also occurs in numerous extrarenal sites in cells that express CYP27B1 like immune cells especially activated macrophages. Immune activation of macrophages with Toll-like receptor (TLR) ligands or IFN-y leads to induction of CYP27B1. As circulating levels of 1,25(OH)2D rise, it suppresses its own production via a negative feedback loop in which the VDR binds to the CYP27B1 promoter to repress its expression(Gombart 2009). Bacillus coagulans is a gram-positive, spore-forming, aerobic, and lactic acidproducing bacteria. In comparison to other lactic acid bacteria, due to its protective spore-like protein coating, it can survive against stomach acid, reach the small intestine, grow, and proliferation(Konuray and Erginkaya 2018). These bacteria produces short-chain fatty acids by secreting coagulin and lactosporin and has antimicrobial activity(Riazi et al. 2009). Researches have shown that cell wall components and intracellular fluid are involved in intestinal inflammation by regulating cytokines, inhibiting reactive oxygen species, and increasing phagocytosis(M Jones et al. 2012; Mittal et al. 2014). They reported that intestinal microbiota plays a primary role in the mucosal surface immune response by balancing Treg and Th17(Wu and Wu 2012). Various mouse models include Experimental autoimmune encephalomyelitis and Cuprizone are widely used to study MS(Ziemssen and Ziemssen 2005). The cuprizone model is applied to study demyelination and remyelination in the corpus callosum. Cuprizone 
induces the death of mature oligodendrocytes, leading to the loss of myelin in the white matter of the brain(Zhen et al. 2017). Therefore, this study aimed to survey the effect of Bacillus coagulans on the improvement of demyelinated mice. Consequently, we performed an analysis of IDO gene expression and serum level of IL-17 and TGF- $\beta$.

\section{Materials And Methods}

\subsection{Animals and Demyelination}

All methods involving animals and their care were according to the Institutional Animal Care and Use Committee recommendations. The practical protocols used in this study were approved by the local animal ethics committee of the Islamic Azad University Science and Research Branch. The medical intervention and treatment procedures were done according to guidelines. Regarding the statistics criteria, the number of mice was the minimum possible. 42 Female C57BL/ 6 mice of 7-8 weeks old with weight ranging from 18 to $20 \mathrm{~g}$ were obtained from Pasteur Institute, Tehran, Iran. The animals had free access to food and water and were maintained on a $12 \mathrm{~h}$ light/dark cycle at room temperature $\left(20-22^{\circ} \mathrm{C}\right)$. According to Zhen et al(Zhen et al. 2017)., toxic demyelination was induced by oral administration containing $0.2 \%(\mathrm{w} / \mathrm{w})$ cuprizone $(\mathrm{CPZ})$.

For oral gavage, $\mathrm{CPZ}$ powder was mixed in $0.5 \%$ carboxymethyl cellulose $(\mathrm{CMC})$ and use vortex shaker to obtain a homogeneous CPZ-CMC suspension. Mice were fed daily by gavage with $10 \mathrm{ml} / \mathrm{kg}$ volume. A stock solution of $0.5 \% \mathrm{CMC}$ was prepared weekly and stored at $4^{\circ} \mathrm{C}$. $\mathrm{CPZ}$ and $\mathrm{CMC}$ suspension were freshly prepared and consumed daily. CMC mixture does not react chemically with the CPZ. When the animal's weight loss was about $20 \%$ of total body weight, CPZ application was halted for a day and continued after the rest day. In all subjects, the maximum time required for rest was only 1 day, and just at the end of the CPZ administration period, some animals lost a maximum of $20 \%$ of their body weight.

\subsection{Experimental Design}

Mice were divided randomly into 6 groups (7 mice in each group). Group 1: normal control (oral administration of distilled water every day for 4 weeks to induce stress same like other groups), group 2: cuprizone control (oral administration of cuprizone $0.2 \% \mathrm{w} / \mathrm{w}$ for 4 weeks), group 3: probiotic control (oral administration of B.coagulans at a concentration of $1 \times 10^{9} \mathrm{CFU} / \mathrm{ml}$ for 4 weeks), group 4: prevention group (oral administration of B.coagulans for 2 weeks then cuprizone for 4 weeks), group 5: Improvement group I (oral administration of cuprizone for 4 weeks then B.coagulans for 4 weeks) and group 6: Improvement group II (oral administration of cuprizone for 4 weeks then B.coagulans and Vitamin D at a concentration of $20 \mathrm{IU} /$ day for 4 weeks). The lyophilized bacterial powder was produced by Tak Gene Zist Pharmaceutical Company, Tehran, Iran. All mice from various groups were examined by behavioral, molecular, and histopathological tests. Every possible effort was made to minimize the number and affliction of used animals.

\subsection{Sampling}


Before being sacrificed, each mouse was in deep anesthesia conditions, and samples were collected by cardiac puncture, and then were investigated by behavioral, molecular, and histopathological tests.

\subsection{Serum Cytokine Levels}

The concentrations of IFN- $\gamma$, IL-17, IL-4, and TGF- $\beta$ in plasma were measured by the enzyme-linked immunosorbent assay(Eckburg et al. 2005) using the mice serum samples. Sandwich ELISA test was performed according to Duo Set kit (R\&D System, USA) instructions. Briefly, the capture antibody was coated in 96 well high binding plates overnight. After washing, wells were blocked with BSA for an hour, and the standard's serial dilution or samples were added to the plate and incubated following another washing stage. Then, the detection antibody was added and incubated for 2 hours. Streptavidin conjugated horseradish peroxidase was applied to complete the reaction. The color reaction was developed by adding the substrate. Finally, the absorbance was estimated by a microplate reader (TECAN, USA) at a wavelength of $405 \mathrm{~nm}$.

\subsection{RNA isolation and Real-Time Polymerase Chain Reaction (RT-PCR)}

The expression of genes was quantified by RT-PCR assay and expressed relative to the expression of the $\beta$-actin gene. Total RNA was extracted with Trizol reagent (Invitrogen) and used as a template to prepare cDNA using M-MLV RTase system (Takara). Gene expression was quantified using the SYBR Green RealTime PCR kit (Toyobo) and the StepOnePlus ${ }^{\mathrm{TM}}$ Real-Time PCR System (Applied Biosystems). The primer sequences used were as follows: 
Table 1

Real-time PCR primers used in gene expression analysis ( $\mathrm{F}$ :

Forward Primer- R: Reverse Primer )

\begin{tabular}{|c|c|}
\hline Gene & Primer \\
\hline \multirow[t]{2}{*}{ AIM2 } & aim2-F: CATGAGAAGTGTGGAGTGAAGT \\
\hline & aim2-R: GTGTCTGGGTTTGGTGGTTG \\
\hline \multirow[t]{2}{*}{ IDO-1 } & ido1-F: GGGGTAGAGAAGGATGTGG \\
\hline & ido1-R: TGAGTAGAGTGTGGTGAAGTG \\
\hline \multirow[t]{2}{*}{$\beta$-Actin } & Bactin-F: TCAGAGCAAGAGAGGCATCC \\
\hline & Bactin-R: GGTCATCTTCTCACGGTTGG \\
\hline \multirow[t]{2}{*}{ CYP27B1 } & cyp27b1-F: GCTGTGATGAAAGAAGTGTTGA \\
\hline & cyp27b1-R: GTGGCATAGTGAGATAGGGAGA \\
\hline \multirow[t]{2}{*}{ NLRP3 } & nlrp3-F: GGACCCACAGTGTAACTTGCAGA \\
\hline & nlrp3-R: AGGCTGCAGTTGTCTAATTCCAG \\
\hline \multirow[t]{2}{*}{ NLRP1 } & nlrp1-F: GGAAACTGAGATGTGATGGTAGA \\
\hline & nlrp1-R: GTTGGATACAGTGAGGTTGGTGT \\
\hline
\end{tabular}

\subsection{Y-Maze Behavioral Test}

The Y-maze is an uncomplicated two-trial memory test for assessing spatial memory. According to Kim et al. 2007, mice were placed in one arm, and the number and sequence of arm entries were recorded for 10 minutes. The percentage of triads in which all three arms were show, i.e., $C A B, A B C$, or $B C A$ but not $B A B$, was recorded as an alternation to evaluate short-term memory. The maze arms were cleaned completely and sprayed with water between tests to remove remaining smells. The alternation score for each mouse was described as the ratio of the actual number of alternation to the possible number of alternation multiplied by 100 as the following equation:

[(number of alternations] / [total arm entries -2$]) \times 100$

The total number of arm entries acted as an indicator of short-term spatial memory.

\subsection{Immunohistochemistry Technique}

Immunohistochemical staining was performed using the avidin-biotin method with an antibody against the MBP to evaluate demyelination.

\subsection{Histological staining}


To evaluate the rate of the remyelination, paraffin sections were stained with Luxol fast blue (LFB). After dehydration, brain sections were incubated with $0.1 \% \mathrm{LFB}$ solution at $60^{\circ} \mathrm{C}$ overnight. Following a rinse with $95 \%, 70 \%$ ethanol, and distilled water, the slides were placed in a separate lithium carbonate solution for $30 \mathrm{~s}$ and then in $70 \%$ ethyl alcohol for another $30 \mathrm{~s}$. The last wash with distilled water was done.

\subsection{Statistical Analysis}

For all experiments, data were analyzed using SPSS 23. A comparison between groups was tested by a two-way analysis of variance, followed by a Tukey Test. Statistical significance is considered when $\mathrm{P}<$ 0.05

\section{Results}

\subsection{B.coagulans Treatment Reduces Pro-Inflammatory Cytokine Expression}

The analyses of anti-inflammatory cytokines are shown in Fig. 1. IL-4's graph shows that there are significant differences between Group 2 as a Cuprizone Control with Group 4, 5, and $6(P=0.000<0.05)$. Also, it shows that there is a significant difference between Group 1 and Group $6(P=0.043<0.05)$ and between Group 3 and Group $4(P=0.022<0.05)$.

According to TFG- $\beta$ 's graph, there are significant differences between Group 2 as a Cuprizone Control with Group 4,5 , and $6(P=0.000<0.05)$. No significant differences were also found in serum TFG- $\beta$ of other experimental groups.

\subsection{B.coagulans Treatment modulates anti-Inflammatory Cytokine Expression}

The analyses of inflammatory cytokines are shown in Fig. 1. The graph of IL-17 demonstrates that there are significant differences between Group 2 as a Cuprizone Control with Group 4, 5, and $6(P=0.000<$ 0.05). Although, there are some significant differences between Group 3 and Group $5(P=0.004<0.05)$ and Group 3 and Group $6(P=0.001<0.05)$.

The graph of IFN- $y$ shows that there are significant differences between Group 2 as a Cuprizone Control with Group 4,5 , and $6(P=0.000<0.05)$. It demonstrates that there is a significant difference between Group 1 and Group $4(P=0.004<0.05)$. This graph also shows that there is a significant difference between Group 3 and Group $4(P=0.010<0.05)$.

\subsection{Analysis of Genes Expression Data in Different groups}

The effects of probiotic administration on the expression of genes are shown in Fig. 2. The relative expression of the $I D O-1$ gene was significantly decreased in the experimental groups compared to the Cuprizone Control Group $(P=0.000<0.05)$. No significant differences were found in $I D O-1$ gene expression between Normal and Probiotic Control Groups with experimental Groups.

The relative expression of CYP27B1gene was significantly decreased in the experimental groups. Group 4 $(P=0.001<0.05)$, Group $5(P=0.000<0.05)$, and Group $6(P=0.000<0.05)$ compared to the Cuprizone 
Control Group.

The relative expression of NLRP1gene was significantly decreased in the experimental groups. Group 4 $(P=0.019<0.05)$, Group $5(P=0.025<0.05)$, and Group $6(P=0.000<0.05)$ compared to the Cuprizone Control Group.

The relative expression of the NLRP3 gene was significantly decreased in the experimental groups. Group $4(P=0.006<0.05)$, Group $5(P=0.013<0.05)$, and Group $6(P=0.008<0.05)$ compared to the Cuprizone Control Group.

The graph of $A I M 2$ shows that there is a significant difference between Group 1 and Group $4(P=0.049<$ 0.05). It also demonstrates that there are some significant differences between Group $4(P=0.002<0.05)$, Group $5(P=0.000<0.05)$, and Group $6(P=0.000<0.05)$ compared to the Cuprizone Control Group.

\subsection{Assessing Short-Term Spatial Memory Using Y-Maze}

According to the obtained results shown in Fig. 3, there is a significant difference between the Cuprizone Control Group and experimental groups $(P=0.000<0.05)$.

\subsection{Confirmation of Demyelination}

The analysis of IHC staining (Fig. 4) demonstrates that there is an acceptable rate of demyelination after 4 weeks of cuprizone administration in 5 and 6 experimental groups in comparison with the normal group (Fig. 5).

\subsection{Rate of Remyelination}

The study of LFB staining indicates that there is an acceptable rate of Myelin Decomposition in experimental groups compared with the cuprizone group (Fig. 6).

\section{Discussion}

Autoimmune diseases can be due to the failure of the tolerance process and the reduction in proliferation of autoreactive T lymphocytes(Rosenblum et al. 2015). Both Th1 and Th17 have an important role in the pathogenesis of this disease. On the other hand, Treg cells are considered important cells in establishing tolerance and modulating the immune response(Tesmer et al. 2008). Dietary changes in the gut microbiota, overuse of antibiotics, and stress are some of the hypotheses that are considered to increase the risk of MS(Langdon et al. 2016). Studies show the MS gut microbiome as having general alterations in specific taxa, some associated with the promotion of inflammatory cytokines and overall inflammation(Kirby and Ochoa-Repáraz 2018). Various important mechanisms underlying the antagonistic effects of probiotics on different microorganisms include the following: modification of the gut microbiota, competitive adherence to the mucosa and epithelium, strengthening of the gut epithelial barrier, and modulation of the immune system to conduct an advantage to the host(Bermudez-Brito et al. 
2012). Probiotics can influence the immune system by metabolites, cell wall components, and DNA. Immune modulatory effects might be even achieved with dead probiotic bacteria or just probioticsderived components like peptidoglycan fragments or DNA(Oelschlaeger 2010). In this study, one of the measured cytokines is IFN-g, which is involved in the pathogenesis of Multiple sclerosis and its level is increased in this disease. In this study, we showed that Bacillus coagulans decreased the IFN-g level, which was consistent with previous studies. Researches have shown that injection of IFN-g to patients with MS causes attacks, and also the IFN-g serum level rising before attacks, indicating that disease activity and recurrence are initially related to Th1 activity(Beck et al. 1988; Becher et al. 1999). On the other hand, IFN-b was found to reduce the secretion of IFN-g from peripheral blood mononuclear cells and improve the patient's clinical condition(Kelleher et al. 2006). Jensen et al. also studied the effect of Bacillus coagulans on the inflammatory properties of IFN-g. Their results showed that these bacteria increased cytokine levels, which contradicts our results(Jensen et al. 2010). During a study on a mouse model, Dargahi et al. showed that Streptococcus thermophilus reduces the secretion of inflammatory cytokines such as IFN-g and IL-1b and continuous use of this probiotic can be effective in controlling and treating autoimmune diseases such as MS(Dargahi et al. 2018). Lee et al. have also shown in the mouse model of colitis after treatment with lactic acid probiotics, the level of IFN-g and IL-1b Dropped(Lee et al. 2017). In a study of the mouse model of EAE by He et al., It was found that the use of oral Lactobacillus reuteri could lead to a decrease in Th1/Th17 cells and related cytokines IFN-g/IL-17. They also showed that the loss of intestinal microbiota diversity that occurred with model induction was regenerated by this probiotic(He et al. 2019). Cosorich et al. found that increased frequency of TH17 cells correlates with high disease activity and with specific alterations of the gut mucosa-associated microbiota in MS patients(Cosorich et al. 2017). Many studies have suggested that probiotics have the ability to inhibit proinflammatory IL-17 production and activation in Th17-related diseases(Owaga et al. 2015; Ortiz et al. 2016). Secher et al. indicated that beneficial traits were associated with a decreased secretion of inflammatory cytokines such as IL-17, IFN- $\gamma$, and TNF- $a$ and an increase in the production of IL-10 as an anti-inflammatory cytokine by autoreactive CD4 + T-cells, CD4 + Foxp3 + in lymph nodes(Secher et al. 2017). Kobayashi et al. in their work indicated that administration of L. casei strain Shirota on the mouse model of EAE upregulated IL-17 and IFN-y in Inguinal lymph nodes cells(Kobayashi et al. 2012). In this research we found that Bacillus coagulans decreased the IL-17 level, which is similar to other studies. Singh et al. in their work demonstrated that VSL\#3 administrations did not lead to any changes in gut flora, but led to a reduction in the frequency of Th17 cells and in the production of pro-inflammatory cytokine on lipopolysaccharide stimulation(Singh et al. 2018). Other studies showed that beneficial probiotic bacteria suppress pathogenic Th17 cells and induce steady-state Th17 cells(Tanabe 2013). Salehipour et al. investigated the effect of $10^{9} \mathrm{CFU} / \mathrm{mL}$ Lactobacillus plantarum A7, Bifidobacterium animalis and a combination of these probiotics in an experimental model of MS. They reported that $L$. plantarum $A 7$ and $B$. animals could ameliorate EAE condition through enhancing the anti-inflammatory cytokines and cell, such as IL-10, IL-4, and TGF- $\beta$, Tregs, CD4+, CD25+, and Foxp3 + in spleen and lymph nodes together with increasing Th2 and Treg in spleen and brain(Salehipour et al. 2017). In present research, we also found Bacillus coagulans has modulatory effect on IL-4 and TGF- $\beta$ and this could confirm the modulating properties of probiotics. Recently, a promising evidence of synergic effects of 
combined supplementation with vitamin $\mathrm{D}$ and probiotics in modulating the gut microbiota and metabolome is emerging(Abboud et al. 2021). Also, numerous observational studies have suggested that there is a correlation between the level of serum vitamin D and MS risk and disease activity(Sintzel et al. 2018). Smolders et al. indicated that there are high levels of CYP27B1 protein in MS lesions and normal appearing white matter, but not in white matter from healthy control subjects(Smolders et al. 2013). We also found that Bacillus coagulans decreased the CYP27b1 gene expression level, which is similar to prior studies. Another of the genes investigated in this study was the IDO-1 gene. Our results showed that cuprizone significantly enhanced the expression in comparison with the control group, and also between treatment groups, consumption of this bacteria significantly reduced the expression in comparison with the cuprizone group. Measurement of IDO gene expression and activity in blood could be a useful marker to monitor the clinical course of RR-MS. Therapeutic interventions modulating IDO activity may be beneficial in MS(Mancuso et al. 2015). Wetzel et al. investigated that Expression of ID01 but not IDO2 was upregulated in the spinal cord during peak symptoms of EAE(Wetzel et al. 2020). Cvijin et al. also showed ID01 activity correlates with loss of barrier-promoting Th17 cells in HIV/simian immunodeficiency virus infection. They proposed that Lactobacillus species inhibit mammalian ID01 and thus may help to preserve Th17 cells during pathogenic SIV infection, providing support for Lactobacillus species as modulators of mucosal immune homeostasis(Vujkovic-Cvijin et al. 2015). Digehsara et al. demonstrated that the administration of Lactobacillus casei decreased the expression of ID01 in mouse model of cuprizone(Digehsara et al. 2021) which is consistent with our results.

Another studied pathway in this article is the Inflammasome pathway which has been tested by the expression of NLRP1, NLRP3, and AIM2. According to our results, there is a significant reduction in expression between treatment groups and the cuprizone group in all three genes. In a study Michailidou et al. showed inhibition of the complement system by PMX205 decreased genes that are keys for inflammasome-related microglial activation: NLRP3, IL-1b, ASC, AIM2, NLR Family Pyrin Domain Containing 1 -NLRP1, NLRC4. PMX205 was also able to reduce IL-1b and Iba1 protein expressions that correlated with reduced demyelination, the extent of axonal injury and improve clinical scores(Olcum et al. 2020). Remyelination as a critical factor in Multiple sclerosis has been targeted in our study. Because of the immune modulatory effects of Bacillus coagulans, we have seen that the rate of remyelination in treatment groups increased significantly in compare to cuprizone control group. McMurran also investigated the potential of a therapeutic probiotic (VSL\#3) to improve remyelination in aged mice. He figured out that In contrast to antibiotic treatment, probiotic administration caused a slight enhancement in the onset of inflammation following focal demyelination(McMurran 2018) and our result is similar. We analyzed spatial memory by Y-Maze so according to the results there was a significant increasing in alternation percentage in treatment groups and cuprizone group. Benton et al proof that the consumption of probiotic-containing yoghurt improved the mood of those whose mood was initially poor(Benton et al. 2007). The results of Ho et al. highlighted a possible antioxidative effect triggered by short-chain fatty acids that contributed to improving the memory and learning abilities following treatment with the probiotic mixture and suggested that probiotics could serve as a therapy to modulate physiological function(Ho et al. 2019). 
In conclusion the data presented in the present study show that oral administration of B.coagulans to the demyelinated mouse model of cuprizone modulates immune responses. This probiotic increases the percentage of alternation behavior and the rate of remyelination. On the other hand, this strain decreases IFN- $\gamma$, IL-17, and modulates IL-4, and TGF- $\beta$ in serum level. Therefore, according to the data of this study, it seems that B.coagulans could suppress Th17 and Th1 responses and help to provide a proper adaptive immune response as well as innate immune response by decreasing the expression of Inflammasome pathway related genes. B.coagulans inhibits the expression of ID01, so it has effects on the Kynurenine pathway by decreases the depletion of Trp and production of neurodegenerative metabolites. As the less Trp enters to Kynurenine pathway, more will be available to get to the serotonin pathway. The synergic effect of combined probiotic and vitamin $D$ creates a proper mix to shows a significant difference between prevention and treatment groups compares to cuprizone control group in CYP27b1 gene expression.

\section{Declarations}

\section{Funding}

No funding was received for conducting this study.

\section{Conflict of Interest}

The authors declare that they have no conflict of interest.

\section{Availability of data and material}

Data and material provide a standardised format.

\section{Code availability}

No code

\section{Authors' contributions}

All authors equally contribute in preparation of this manuscript.

\section{Ethical approval and consent to participate}

All applicable international, national, and/or institutional guidelines for the care and use of animals were followed by the authors.

\section{Consent for publication}

All authors consent for publication.

\section{Acknowledgements}


No acknowledgements

\section{References}

1. Abboud M, Rizk R, AlAnouti F, et al (2021) The Health Effects of Vitamin D and Probiotic CoSupplementation: A Systematic Review of Randomized Controlled Trials. Nutrients 13:111

2. Bar-Or A, Rieckmann P, Traboulsee A, Yong VW (2011) Targeting Progressive Neuroaxonal Injury. CNS Drugs 25:783-799

3. Becher B, Giacomini PS, Pelletier D, et al (1999) Interferon-y secretion by peripheral blood T-cell subsets in multiple sclerosis: Correlation with disease phase and interferon- $\beta$ therapy. Ann Neurol Off J Am Neurol Assoc Child Neurol Soc 45:247-250

4. Beck J, Rondot P, Catinot $L$, et al (1988) Increased production of interferon gamma and tumor necrosis factor precedes clinical manifestation in multiple sclerosis: do cytokines trigger off exacerbations? Acta Neurol Scand 78:318-323

5. Belkaid Y, Hand TW (2014) Role of the microbiota in immunity and inflammation. Cell 157:121-141

6. Benton D, Williams C, Brown A (2007) Impact of consuming a milk drink containing a probiotic on mood and cognition. Eur J Clin Nutr 61:355-361

7. Bermudez-Brito M, Plaza-Díaz J, Muñoz-Quezada S, et al (2012) Probiotic mechanisms of action. Ann Nutr Metab 61:160-174

8. Cosorich I, Dalla-Costa G, Sorini C, et al (2017) High frequency of intestinal TH17 cells correlates with microbiota alterations and disease activity in multiple sclerosis. Sci Adv 3:e1700492

9. Dargahi N, Johnson J, Donkor O, et al (2018) Immunomodulatory effects of Streptococcus thermophilus on U937 monocyte cell cultures. J Funct Foods 49:241-249. https://doi.org/https://doi.org/10.1016/j.jff.2018.08.038

10. Digehsara SG, Esfandiari B, Karim E, et al (2021) Effects of Lactobacillus casei Strain T2 (IBRCM10783) on the Modulation of Th17/Treg and Evaluation of miR-155, miR-25, and ID0-1 Expression in a Cuprizone-Induced C57BL/ 6 Mouse Model of Demyelination. Inflammation 44:334-343

11. Duhen R, Glatigny S, Arbelaez C, et al (2013) Pathogenicity of IFN- $\gamma+$ Th17 cells is independent of Tbet (P4128)

12. Eckburg PB, Bik EM, Bernstein CN, et al (2005) Diversity of the human intestinal microbial flora. Science (80- ) 308:1635-1638

13. Gombart AF (2009) The vitamin D-antimicrobial peptide pathway and its role in protection against infection. Future Microbiol 4:1151-1165

14. Goodin DS (2016) The epidemiology of multiple sclerosis: insights to a causal cascade. Handb Clin Neurol 138:173-206

15. Govindarajan V, de Rivero Vaccari JP, Keane RW (2020) Role of inflammasomes in multiple sclerosis and their potential as therapeutic targets. J Neuroinflammation 17:1-15 
16. He B, Hoang TK, Tian X, et al (2019) Lactobacillus reuteri Reduces the Severity of Experimental Autoimmune Encephalomyelitis in Mice by Modulating Gut Microbiota . Front. Immunol. 10:385

17. Ho S-T, Hsieh Y-T, Wang S-Y, Chen M-J (2019) Improving effect of a probiotic mixture on memory and learning abilities in d-galactose-treated aging mice. J Dairy Sci 102:1901-1909

18. Høglund RA, Maghazachi AA (2014) Multiple sclerosis and the role of immune cells. World J Exp Med 4:27

19. Hughes RL (2020) A review of the role of the gut microbiome in personalized sports nutrition. Front Nutr 6:191

20. Jensen GS, Benson KF, Carter SG, Endres JR (2010) GanedenBC $30^{\mathrm{TM}}$ cell wall and metabolites: antiinflammatory and immune modulating effects in vitro. BMC Immunol 11:1-14

21. Kelleher $P$, Goodsall $A$, Mulgirigama $A$, et al (2006) Interferon- $y$ therapy in two patients with progressive chronic pulmonary aspergillosis. Eur Respir J 27:1307-1310

22. Kirby TO, Ochoa-Repáraz J (2018) The gut microbiome in multiple sclerosis: a potential therapeutic avenue. Med Sci 6:69

23. Kobayashi T, Suzuki T, Kaji R, et al (2012) Probiotic upregulation of peripheral IL-17 responses does not exacerbate neurological symptoms in experimental autoimmune encephalomyelitis mouse models. Immunopharmacol Immunotoxicol 34:423-433

24. König J, Wells J, Cani PD, et al (2016) Human intestinal barrier function in health and disease. Clin Transl Gastroenterol 7:e196

25. Konuray G, Erginkaya Z (2018) Potential use of Bacillus coagulans in the food industry. Foods 7:92

26. Langdon A, Crook N, Dantas G (2016) The effects of antibiotics on the microbiome throughout development and alternative approaches for therapeutic modulation. Genome Med 8:39. https://doi.org/10.1186/s13073-016-0294-z

27. Lee PW, Severin ME, Lovett-Racke AE (2017) TGF- $\beta$ regulation of encephalitogenic and regulatory $T$ cells in multiple sclerosis. Eur $\mathrm{J}$ Immunol 47:446-453

28. Lee YK, Mazmanian SK (2010) Has the microbiota played a critical role in the evolution of the adaptive immune system? Science (80-) 330:1768-1773

29. Lovelace MD, Varney B, Sundaram G, et al (2016) Current evidence for a role of the kynurenine pathway of tryptophan metabolism in multiple sclerosis. Front. Immunol. 7

30. M Jones R, W Mercante J, S Neish A (2012) Reactive oxygen production induced by the gut microbiota: pharmacotherapeutic implications. Curr Med Chem 19:1519-1529

31. Mancuso R, Hernis A, Agostini S, et al (2015) Indoleamine 2, 3 dioxygenase (IDO) expression and activity in relapsing-remitting multiple sclerosis. PLoS One 10:e0130715

32. McMurran CE (2018) CNS Remyelination and the Gut Microbiota

33. Mittal M, Siddiqui MR, Tran K, et al (2014) Reactive oxygen species in inflammation and tissue injury. Antioxid Redox Signal 20:1126-1167

34. Oelschlaeger TA (2010) Mechanisms of probiotic actions-a review. Int J Med Microbiol 300:57-62 
35. Olcum M, Tastan B, Kiser C, et al (2020) Microglial NLRP3 inflammasome activation in multiple sclerosis. Adv Protein Chem Struct Biol 119:247-308

36. Ortiz AM, Klase ZA, DiNapoli SR, et al (2016) IL-21 and probiotic therapy improve Th17 frequencies, microbial translocation, and microbiome in ARV-treated, SIV-infected macaques. Mucosal Immunol 9:458-467. https://doi.org/10.1038/mi.2015.75

37. Ostadmohammadi V, Jamilian M, Bahmani F, Asemi Z (2019) Vitamin D and probiotic cosupplementation affects mental health, hormonal, inflammatory and oxidative stress parameters in women with polycystic ovary syndrome. J Ovarian Res 12:1-8

38. Owaga E, Hsieh R-H, Mugendi B, et al (2015) Th17 Cells as Potential Probiotic Therapeutic Targets in Inflammatory Bowel Diseases. Int J Mol Sci 16:20841-20858.

https://doi.org/10.3390/ijms160920841

39. Riazi S, Wirawan RE, Badmaev V, Chikindas ML (2009) Characterization of lactosporin, a novel antimicrobial protein produced by Bacillus coagulans ATCC 7050. J Appl Microbiol 106:1370-1377

40. Rosenblum MD, Remedios KA, Abbas AK (2015) Mechanisms of human autoimmunity. J Clin Invest 125:2228-2233. https://doi.org/10.1172/JCl78088

41. Salehipour Z, Haghmorad D, Sankian M, et al (2017) Bifidobacterium animalis in combination with human origin of Lactobacillus plantarum ameliorate neuroinflammation in experimental model of multiple sclerosis by altering CD4+ T cell subset balance. Biomed Pharmacother 95:1535-1548

42. Schattling B, Eggert B, Friese MA (2014) Acquired channelopathies as contributors to development and progression of multiple sclerosis. Exp Neurol 262:28-36

43. Secher T, Kassem S, Benamar M, et al (2017) Oral Administration of the Probiotic Strain Escherichia coli Nissle 1917 Reduces Susceptibility to Neuroinflammation and Repairs Experimental Autoimmune Encephalomyelitis-Induced Intestinal Barrier Dysfunction. Front Immunol. 2017; 8: 1096

44. Singh A, Sarangi AN, Goel A, et al (2018) Effect of administration of a probiotic preparation on gut microbiota and immune response in healthy women in India: an open-label, single-arm pilot study. BMC Gastroenterol 18:85. https://doi.org/10.1186/s12876-018-0819-6

45. Sintzel MB, Rametta M, Reder AT (2018) Vitamin D and multiple sclerosis: a comprehensive review. Neurol Ther 7:59-85

46. Smolders J, Schuurman KG, Strien ME van, et al (2013) Expression of vitamin D receptor and metabolizing enzymes in multiple sclerosis-affected brain tissue. J Neuropathol Exp Neurol 72:91105

47. Tanabe S (2013) The Effect of Probiotics and Gut Microbiota on Th17 Cells. Int Rev Immunol 32:511-525. https://doi.org/10.3109/08830185.2013.839665

48. Tesmer LA, Lundy SK, Sarkar S, Fox DA (2008) Th17 cells in human disease. Immunol Rev 223:87113. https://doi.org/10.1111/j.1600-065X.2008.00628.x

49. Usami M, Miyoshi M, Yamashita H (2015) Gut microbiota and host metabolism in liver cirrhosis. World J Gastroenterol 21:11597 
50. Vujkovic-Cvijin I, Swainson LA, Chu SN, et al (2015) Gut-Resident Lactobacillus Abundance Associates with ID01 Inhibition and Th17 Dynamics in SIV-Infected Macaques. Cell Rep 13:15891597. https://doi.org/10.1016/j.celrep.2015.10.026

51. Wetzel LA, Hurtado M, Kaswan ZAM, et al (2020) Deletion of indoleamine 2, 3 dioxygenase (Ido) 1 but not Ido2 exacerbates disease symptoms of MOG35-55-induced experimental autoimmune encephalomyelitis. Brain, Behav Immunity-Health 7:100116

52. Wu H-J, Wu E (2012) The role of gut microbiota in immune homeostasis and autoimmunity. Gut Microbes 3:4-14

53. Yan F, Polk DB (2011) Probiotics and immune health. Curr Opin Gastroenterol 27:496

54. Yu X, Yu M, Liu Y, Yu S (2016) TRP channel functions in the gastrointestinal tract. In: Seminars in immunopathology. Springer, pp 385-396

55. Zhen W, Liu A, Lu J, et al (2017) An alternative cuprizone-induced demyelination and remyelination mouse model. ASN Neuro 9:1759091417725174

56. Ziemssen T, Ziemssen $F$ (2005) The role of the humoral immune system in multiple sclerosis (MS) and its animal model experimental autoimmune encephalomyelitis (EAE). Autoimmun Rev 4:460467

\section{Figures}



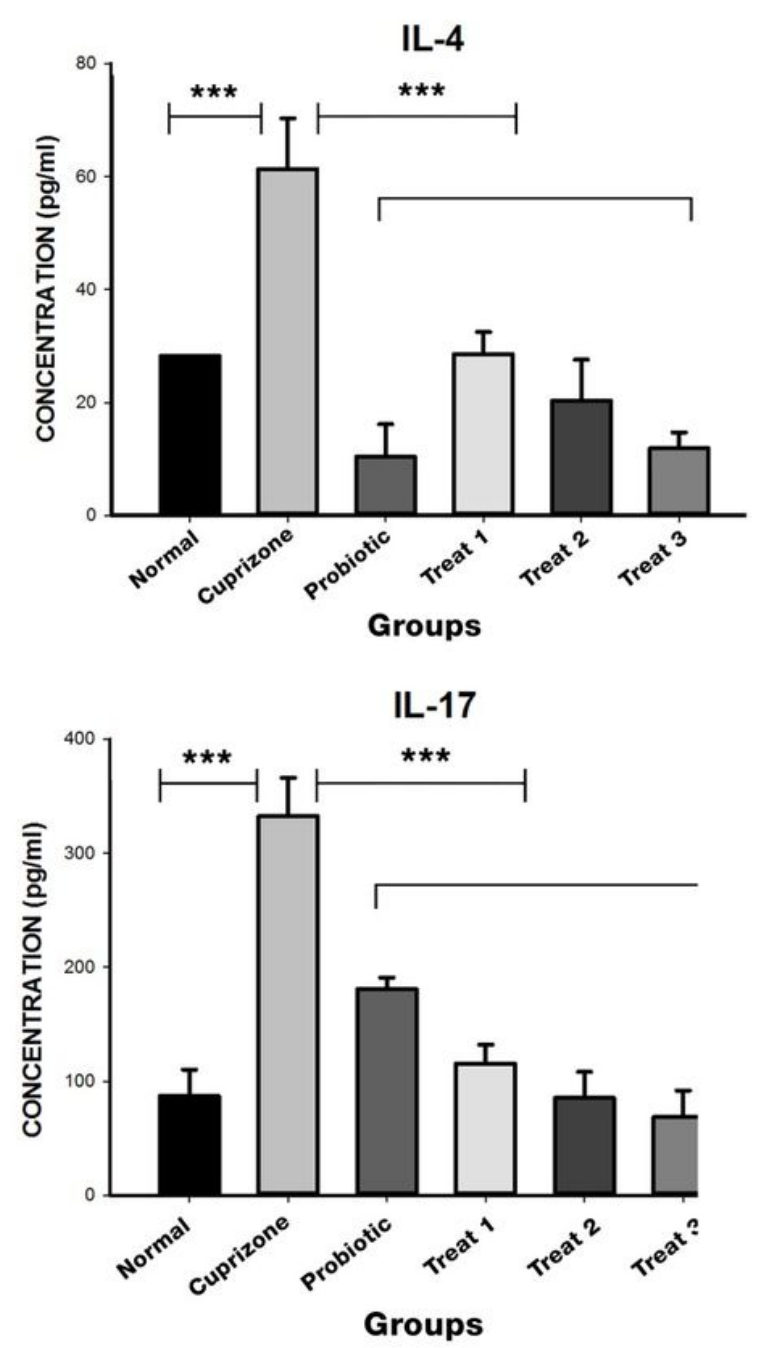

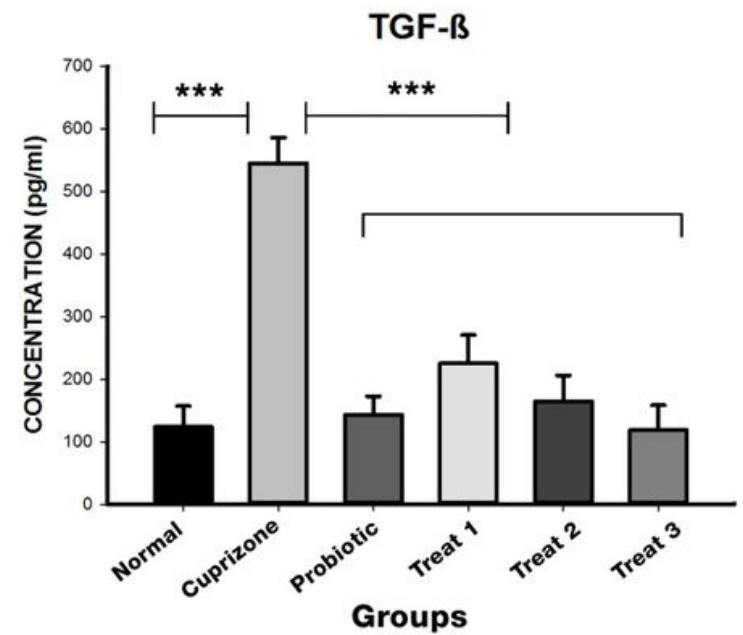

IFN- $\gamma$

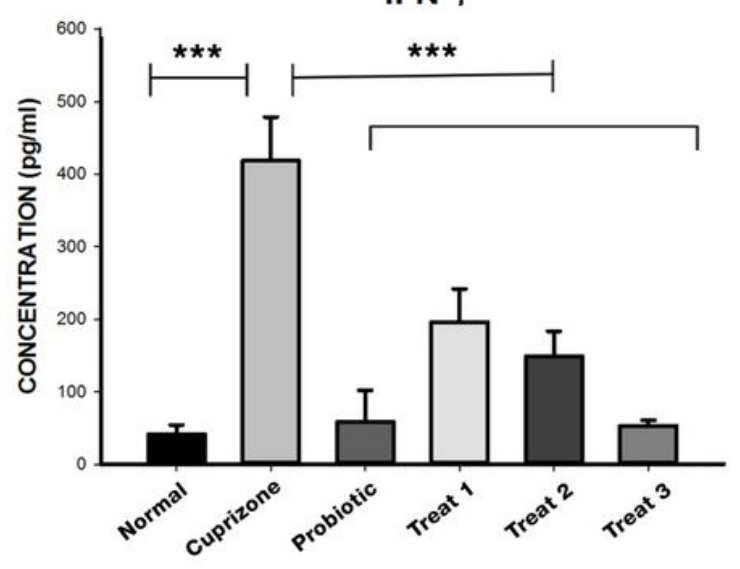

Groups

Figure 1

IL-4, TGF-beta, IL-17, IFN-gamma cytokine level in the serum of demyelinated mouse model of cuprizone. Asterisks are showing significant differences between Bacillus coagulans administration groups and Normal Control, Cuprizone Control, and Probiotic Control. 
IDO-1
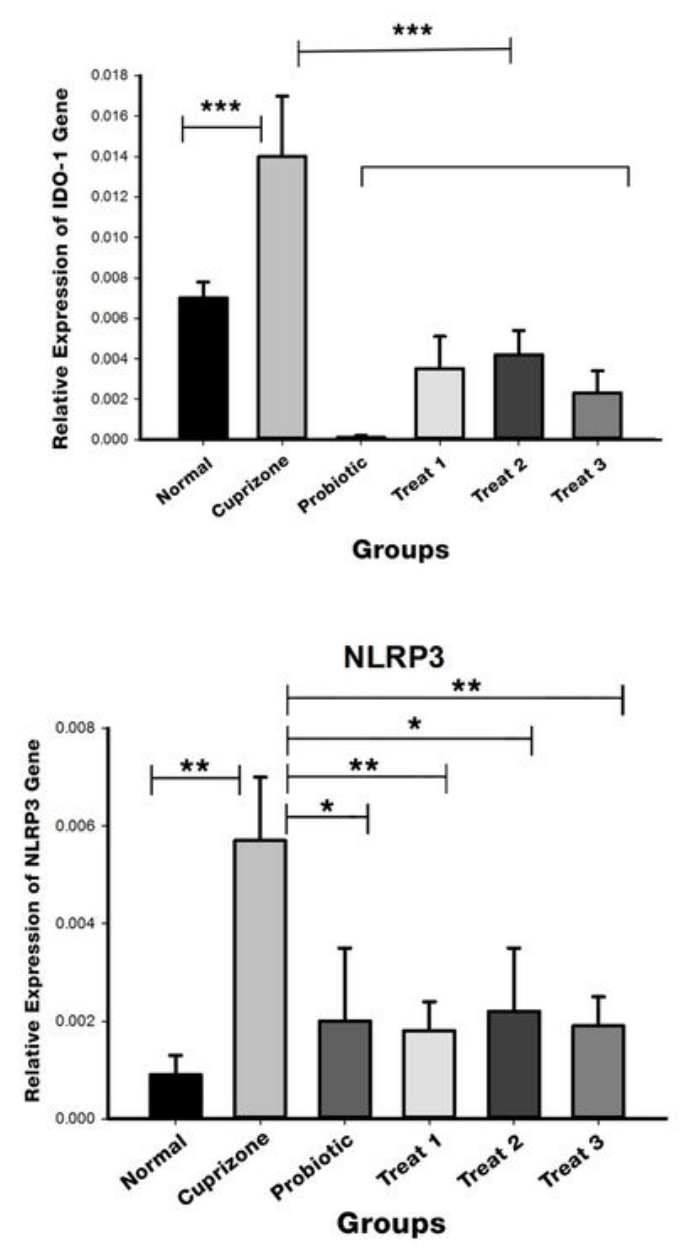

CYP27b1
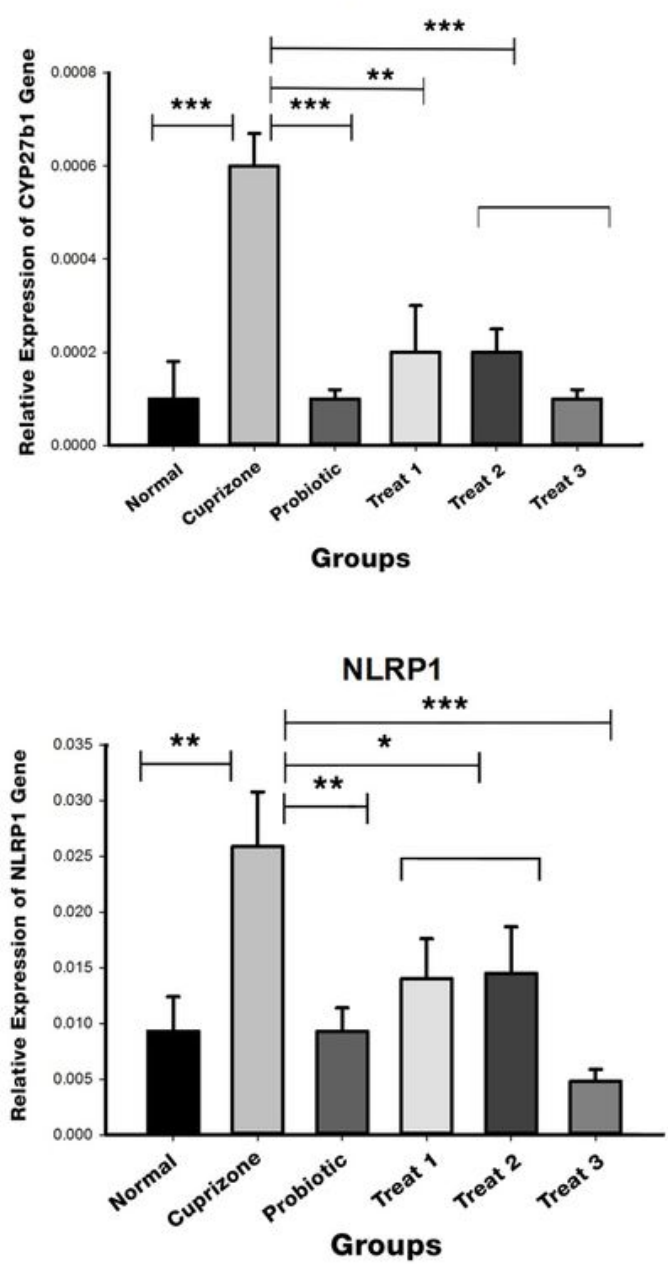

AIM2

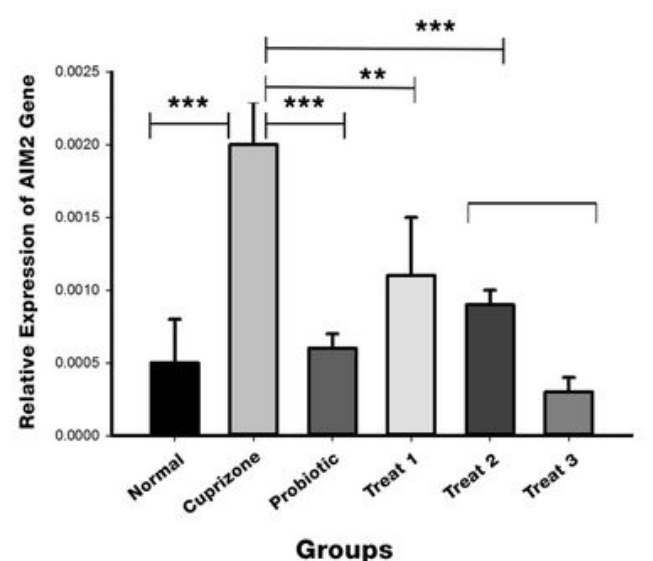

Groups

\section{Figure 2}

Cyp27b1, NLRP1, AIM2, NLRP3, ID0-1 gene expression in the peripheral blood of the demyelinated mouse model of cuprizone. Data were normalized based on $\beta$-actin gene expression and presented as normalized gene expression ( $2-\Delta \Delta C T ; n=5)$. Asterisks are showing significant differences between Bacillus coagulans administration groups and Normal Control, Cuprizone Control, and Probiotic Control. 


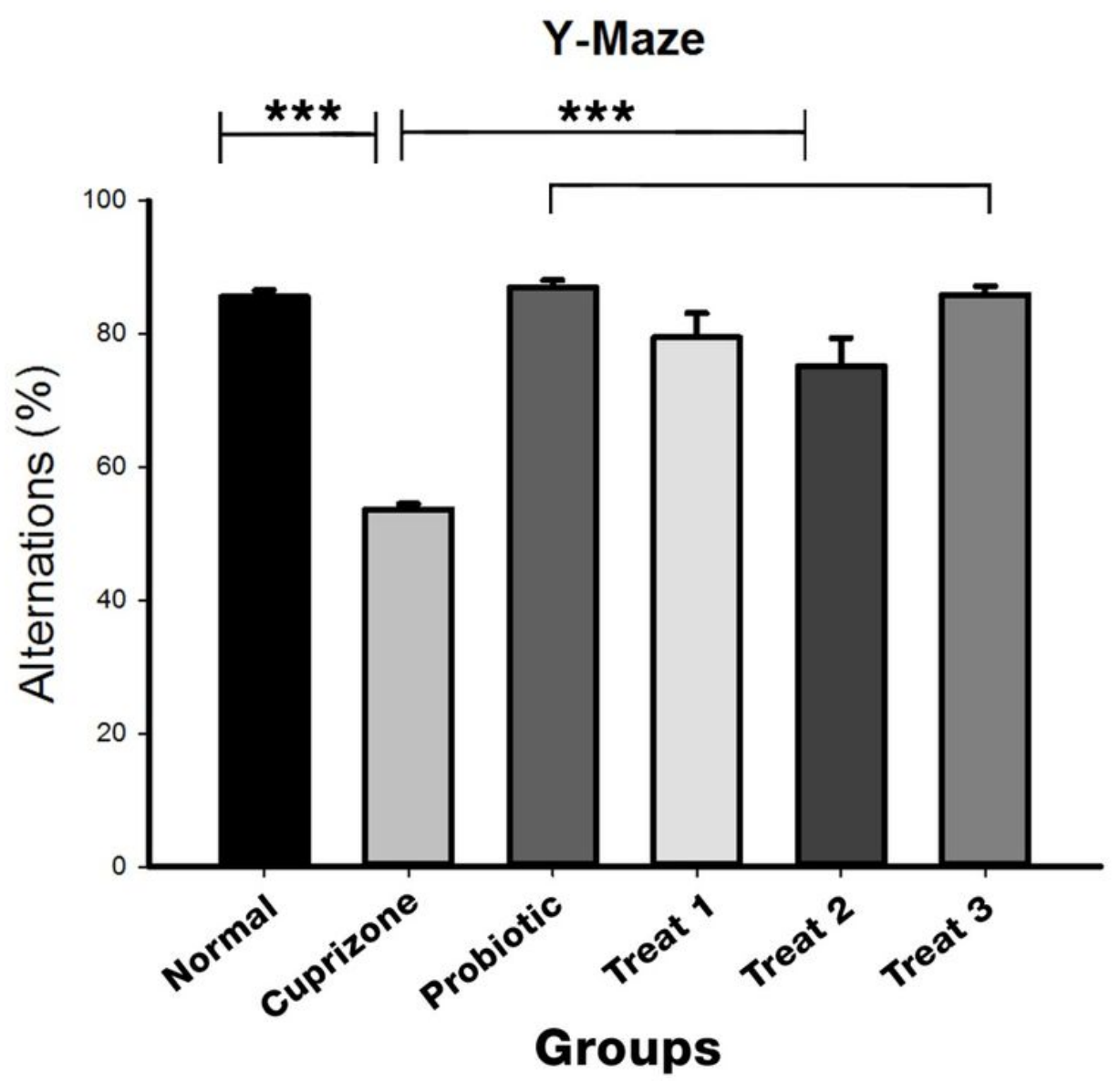

Figure 3

Bacillus coagulans administration enhances spatial cognition in a demyelinated mouse model of cuprizone. The mean alternative rate in the Y-Maze. Asterisks are showing significant differences between Bacillus coagulans administration groups and Normal Control, Cuprizone Control, and Probiotic Control. 

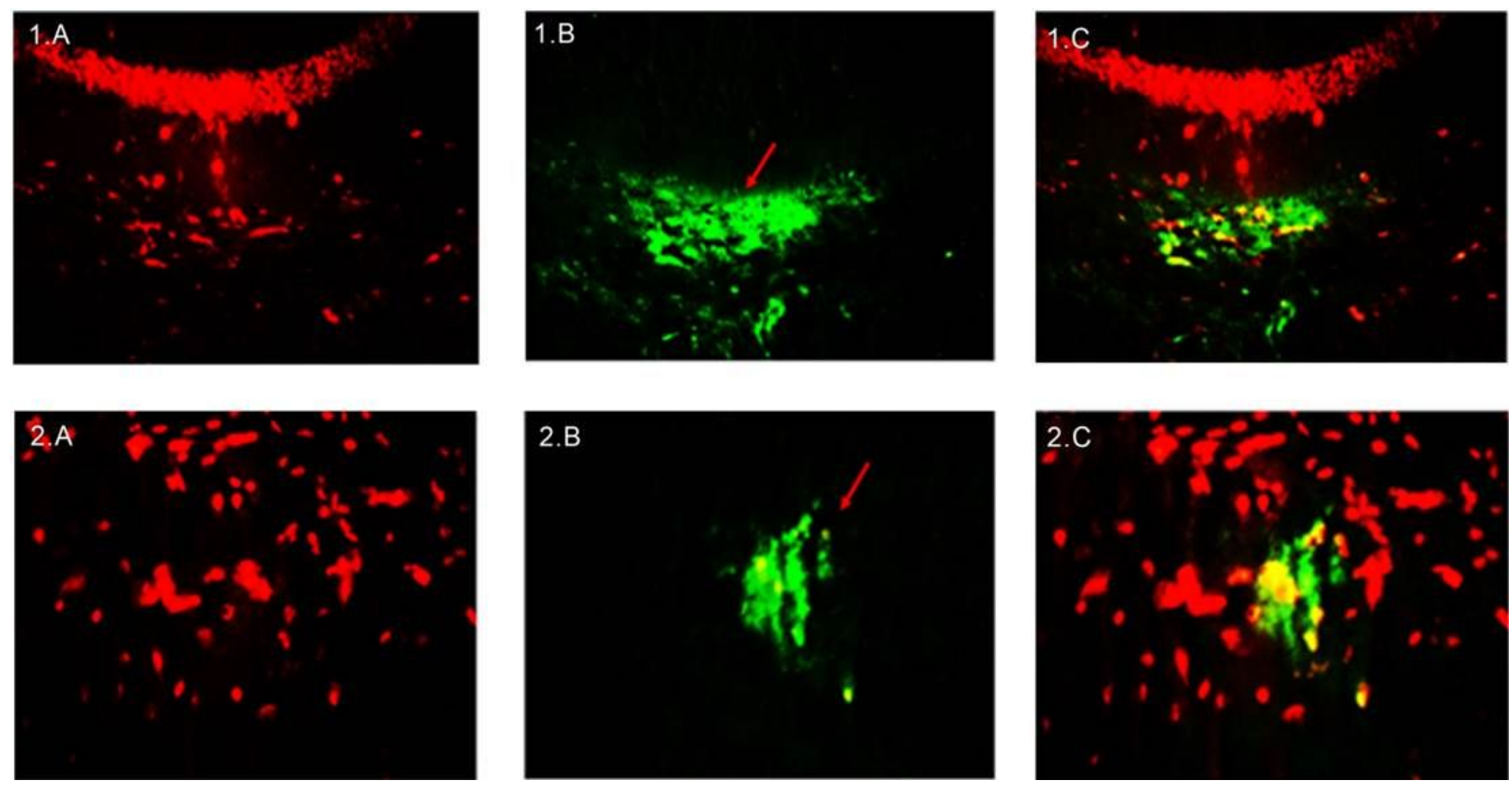

Figure 4

$\mathrm{IHC}$ staining indicated the rate of myelin in the Normal group (magnification:400X) 1.A.Nuclei stained by Propidium iodide 1.B.Primary antibody 1.C.Merge 2.IHC staining indicated the rate of myelin in Cuprizone administration groups after 4 weeks (magnification:400X) 2.A.Nuclei stained by Propidium iodide 2.B.Primary antibody to 2.C.Merge 


\section{IHC}

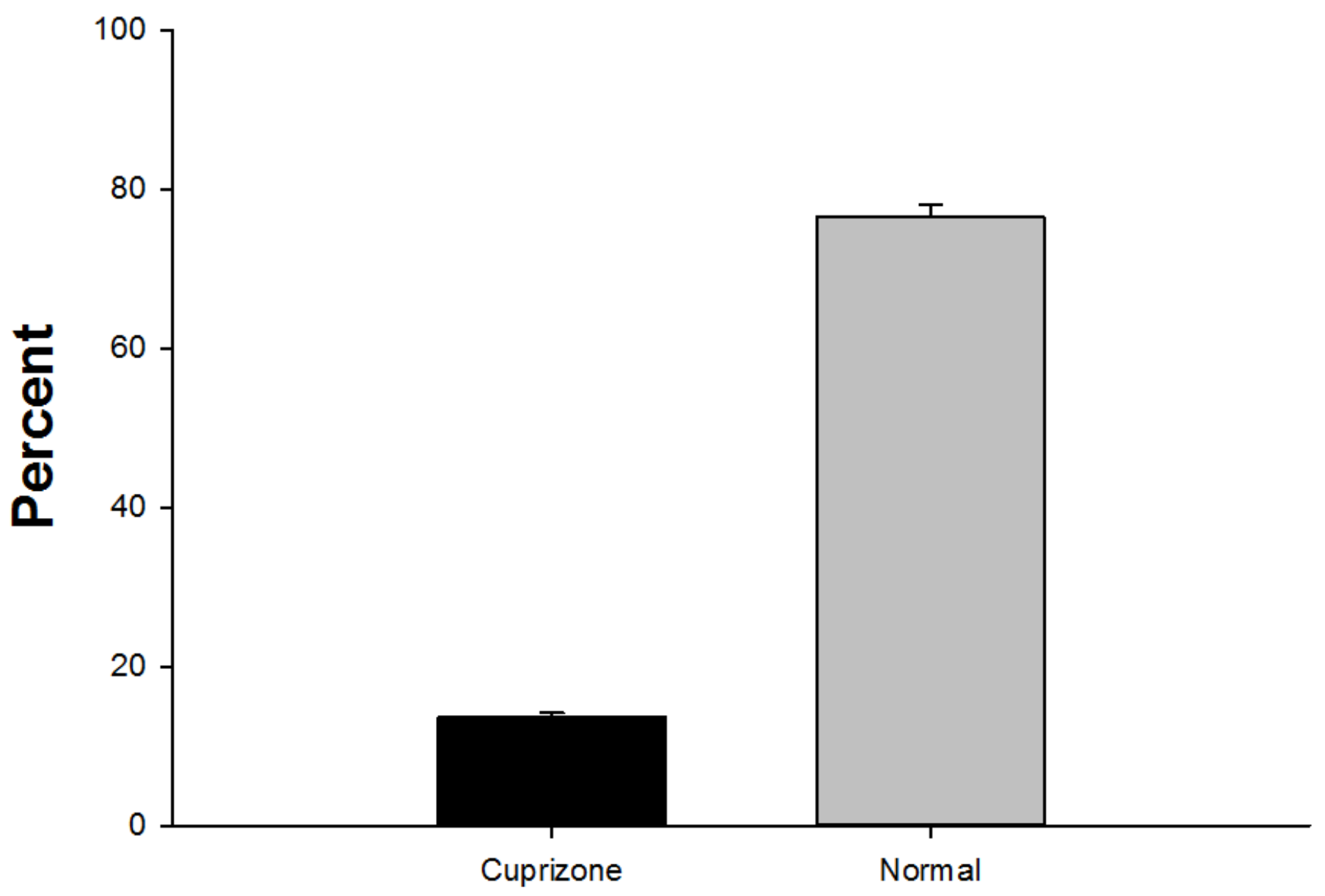

Figure 5

Myelin decomposition Analysis after 4 weeks administration of cuprizone. Asterisks are showing significant differences between Cuprizone Control and Normal Control. 


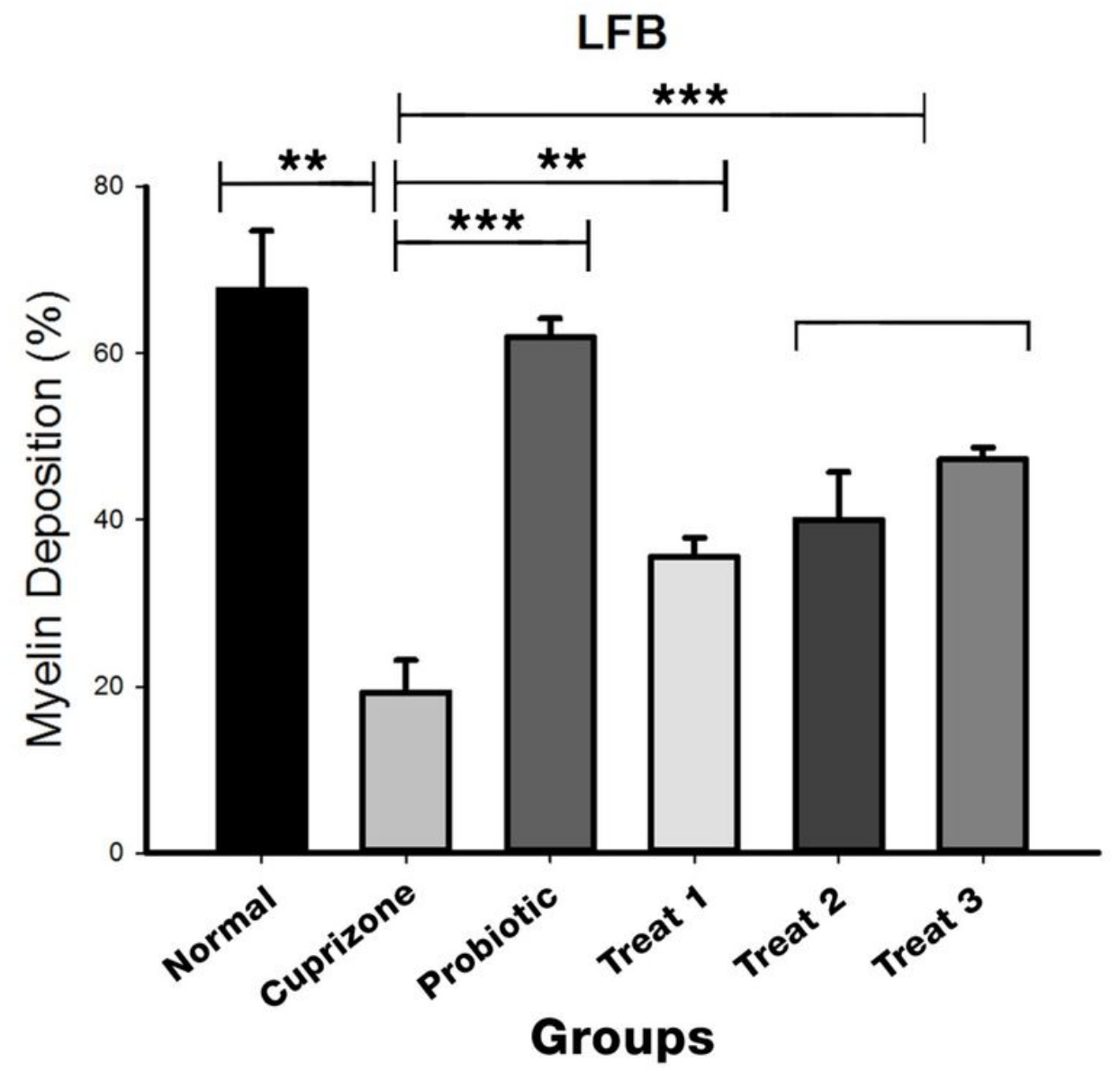

Figure 6

Analysis of the rate of remyelination by Luxol Fast Blue staining in C57bl/ 6 mice treated by Bacillus coagulans. Asterisks are showing significant differences between Bacillus coagulans administration groups and Normal Control, Cuprizone Control, and Probiotic Control. 